\title{
Trypanosoma rangeli sialidase lacks trans-sialidase activity
}

\author{
Lain C. Pontes-de-Carvalho ${ }^{*, a, b}$, Stephen Tomlinson ${ }^{a}$ and Victor Nussenzweig ${ }^{a}$ \\ ${ }^{a}$ Michael Heidelberger Division of Immunology, Department of Pathology and Kaplan Cancer Center, New York University \\ Medical Center, New York, NY 10016, USA; and ${ }^{\mathrm{b}}$ Centro de Pesquisas Goncalo Moniz, Fundacão Oswaldo Cruz, Salvador, BA, \\ Brazil
}

(Received 21 April 1993; accepted 14 July 1993)

\begin{abstract}
Extracts and tissue culture supernatants of axenic forms of $T$. rangeli were assayed for the presence of sialidase and transsialidase activities. Using sialyl( $\alpha 2-3)$ lactose, sialyl $(\alpha 2-6)$ lactose, poly $(\alpha 2-8) N$-acetylneuraminic acid, fetuin and 4methylumbelliferyl- $N$-acetylneuraminic acid as sialic acid donors, and lactose as a sialic acid acceptor, no trans-sialidase activity was detected. Nevertheless, $T$. rangeli lysates and culture supernatants contain a sialidase that hydrolyzes sialyl $(\alpha 2-$ 3)lactose, and much less efficiently sialyl( $\alpha 2-6)$ lactose, but not poly $(\alpha 2-8) N$-acetylneuraminic acid. $T$. cruzi trans-sialidase hydrolyzed only sialyl $(\alpha 2-3)$ lactose under the same conditions. The $T$. rangeli and the $T$. cruzi enzymes differ antigenically and in their $\mathrm{pH}$ optimum for hydrolase activity.
\end{abstract}

Key words: Trypanosoma rangeli; Sialidase; Neuraminidase; Trans-sialidase

\section{Introduction}

Pereira and coworkers have documented the presence of sialidases in Trypanosoma cruzi [1] and Trypanosoma rangeli [2], and suggested that this enzyme plays a role in modulating the initial interactions of $T$. cruzi trypomastigotes with host cells, and in the pathogenesis of Chagas' disease [1]. More recently, the T. cruzi enzyme was shown to function principally as a trans-sialidase (TS) [3-5]: it transfers $\alpha(2,3)$ linked sialic acid to terminal $\beta$-linked galactopyranosyl residues [6-8, reviewed in 9,10]. An enzyme with similar donor and acceptor specificities was also described in procyclics of T. brucei, an insect form of the parasite [11]. The $T$. cruzi TS appears to be involved in the

\footnotetext{
${ }^{\mp}$ Corresponding author. Tel.: 212-2638514; Fax 212-2638179.

Abbreviations: Con A, concanavalin A; HEPES, 4-(2-hydroxyethyl)-1-piperazineethanesulfonic acid; MES, 2-( $N$-morpholino)ethanesulfonic acid; NP-40, Nonidet P-40; Tris, tris(hydroxymethyl)aminomethane; TS, trans-sialidase.
}

attachment to and penetration of host cells by trypomastigotes, presumably through the recognition/generation of sialylated ligands on the surface membrane of target cells and of the parasite $[6,12,13]$. Other findings suggest that the $T$. cruzi TS facilitates the escape of trypomastigotes from the phagocytic vacuoles into the cytoplasm of the host cells [14].

The two trypanosomes $T$. cruzi and $T$. rangeli share several antigens $[15,16]$ and are transmitted by blood-sucking insect vectors of the same genera, Triatoma, Rhodnius and Panstrongylus [17-19]. However, the two parasites differ widely in their development in the mammalian host. $T$. cruzi is pathogenic and multiplies intracellularly $[17,18]$, whereas $T$. rangeli does not invade cells in vitro. Very few $T$. rangeli are ever found in the circulation of infected experimental animals or humans [19], and infection is asymptomatic. Since $T$. cruzi interactions with host cells appear to involve TS-mediated recognition events, here we compare the properties of the sialidase/TS of T.cruzi with the $T$. rangeli enzyme. 
Materials and Methods

Parasites and lysates. Two different $T$. rangeli strains were studied: SC-58 [20], kindly supplied by Dr. M. Deane, Departamento de Protozoologia, Fundação Oswaldo Cruz, Rio de Janeiro, Brazil, and ATCC 30032 (American Type Culture Collection, Rockville, MD), kindly supplied by Dr. J.E. Donelson, Department of Biochemistry, University of Iowa, USA. Parasites were cultured in liver infusiontryptose medium (Difco Laboratories, Detroit, MI) [21] containing $10 \%$ fetal calf serum (Hyclone Laboratories Inc., Logan, UT) at $27^{\circ} \mathrm{C}$. ATCC $30032 T$. rangeli cultures yielded $50 \%$ to $60 \%$ long epimastigotes at high parasite density, whereas SC-58 $T$. rangeli cultures yielded $85 \%$ to $95 \%$ long epimastigotes at high parasite density. The Y strain [22] of $T$. cruz $i$ trypomastigotes were obtained by infecting LLC-MK2 cells (CCL-7; American Type Culture Collection) [6]. Epimastigotes of $T$. cruzi were obtained in liver infusiontryptose medium at $27^{\circ} \mathrm{C}$. T. brucei brucei procyclic trypomastigotes (TREU 667-stock) [23], kindly supplied by Dr. E.J. Bienen, Department of Medical and Molecular Parasitology, New York University Medical Center, New York, USA, were grown in buffered semi-defined medium containing $10 \%$ fetal calf serum at $27^{\circ} \mathrm{C}$ [24]. Parasite lysates were prepared by incubating $10^{9}$ washed parasites with $1 \mathrm{ml}$ of $50 \mathrm{mM}$ tris(hydroxymethyl)aminomethane (Tris)- $\mathrm{HCl}, \mathrm{pH} \mathrm{7.4,} \mathrm{containing}$ $1.5 \%$ Nonidet P-40 (NP-40), $1 \mathrm{mM}$ phenylmethanesulfonylfluoride, $5 \mu \mathrm{g} \mathrm{ml}^{-1}$ of antipain, pepstatin and leupeptin (Sigma Chemical Co., St. Louis, MO) at $4^{\circ} \mathrm{C}$. The lysates were cleared by centrifugation at $10000 \mathrm{~g}$ for $5 \mathrm{~min}$ at $4^{\circ} \mathrm{C}$.

Enzymatic assays and sialic acid measurement. Trans-sialidase activity in $T$. rangeli lysates and supernatants was usually assayed by incubation with $1 \mathrm{mM} \alpha(2-3)$-sialyllactose (Boehringer Mannheim Biochemicals, Indianapolis, IN) and $7.2 \mathrm{mM}$ [D-glucose- $\left.1-{ }^{14} \mathrm{C}\right]$-lactose $\left(60 \mathrm{Ci} \mathrm{mol}{ }^{-1}\right.$; Amersham Corp., Arlington Heights, IL), diluted in a final volume of $50 \mu \mathrm{l}$ of $50 \mathrm{mM}$ 4-(2-hydroxyethyl)-1-piperazineeth- anesulfonic acid (HEPES; Sigma), pH 7, for 50 min at room temperature [6]. Under these conditions the trans-sialidases of $T$. cruzi and $T$. brucei transfer sialyl residues from the sialyllactose to the radioactive lactose $[6,11]$. The generated radioactive sialyllactose is subsequently separated from free lactose by retention on $0.5 \mathrm{ml}$ QAE-Sephadex A50 columns. Results are expressed as cpm eluted from the columns with $0.5 \mathrm{ml}$ of $1 \mathrm{M}$ ammonium formate [6]. In some assays sialyl $(\alpha 2-6)$ lactose (Oxford Glycosystems Inc., Rosedale, NY), $\operatorname{poly}(\alpha 2-8) N$ acetylneuraminic acid (colominic acid), fetuin or 4-methylumbelliferyl- $N$-acetylneuraminic acid (Sigma) were substituted for the sialyl $(\alpha 2$ 3)lactose.

Sialidase activity was assayed using 4methylumbelliferyl- $N$-acetylneuraminic acid as substrate, and measuring the fluorescent product 4-methylumbelliferone at $420 \mathrm{~nm}$, after excitation at $365 \mathrm{~nm}$, in a Titertek Fluoroskan II (Flow Laboratories Inc., McLean, VA). Results are expressed in fluorescence units [3]. In general, the samples were assayed in $50 \mathrm{mM}$ HEPES, pH 6.6, but buffers with different $\mathrm{pH}$, identified in the legend of Fig. 4, were also used.

The comparison between the substrate specificities of the $T$. rangeli, $T$. cruzi and Vibrio cholerae sialidases was performed as follows. A $T$. rangeli culture supernatant was extensively dialyzed against $0.15 \mathrm{M}$ phosphatebuffered saline, $\mathrm{pH} \mathrm{7.2,} \mathrm{in} \mathrm{order} \mathrm{to} \mathrm{remove} \mathrm{free}$ sialic acid molecules. Ten microliters of the dialyzed supernatant, $10 \mu \mathrm{l}$ of a solution of $T$. cruzi trans-sialidase purified by affinity chromatography on concanavalin A (Con A)Sepharose (Pharmacia-LKB Biotechnology Inc., Piscataway, NJ) [6], or $10 \mu \mathrm{l}$ of $4 \mathrm{U}$. $\mathrm{ml}^{-1} V$. cholerae sialidase (Boehringer Mannheim Biochemicals) were incubated with $40 \mathrm{ml}$ of $7.5 \mathrm{mM}$ sialyl $(\alpha 2-3)$ lactose, sialyl $(\alpha 2-$ 6)lactose, or a pentamer of ( $\alpha 2-8)$-linked $N$ acetylneuraminic acid (EY Laboratories Inc., San Mateo, CA). The incubation was carried out at the optimum $\mathrm{pH}$ for each enzyme, i.e., in $50 \mathrm{mM}$ 2-( $N$-morpholino)ethanesulfonic acid (MES; Sigma), pH 5.5 (T. rangeli supernatant and $V$. cholerae sialidase) or in $50 \mathrm{mM}$ HEPES, 
pH 6.6 (T. cruzi TS). The degree of purity of these saccharides was assessed by thin-layer chromatography on silica gel 60 plates (Macherey-Nagel, Düren, Germany) using ethanol $/ n$-butanol/pyridine/water/acetic acid [100:10:10:30:3 (v/v)] [6]. The amount of free sialic acid present in $7 \mu$ l of the reaction mixtures was measured after $0.8,3.5,20$ or 72 $\mathrm{h}$ of incubation at room temperature by the thiobarbituric acid method [25], and expressed as nmol of free sialic acid present in the total reaction mixture. The HPLC-thiobarbituric acid method [26] was used to investigate the presence of sialic acid in $T$. rangeli. Parasites were washed six times with cold DME containing $0.05 \%$ bovine serum albumin (Ultrapure, Boehringer Mannheim Biochemicals) and incubated in $0.1 \mathrm{M}$ sulfuric acid for $1 \mathrm{~h}$ at $80^{\circ} \mathrm{C}$ before the assay.
Fractionation of $T$. rangeli lysates. Lysate and culture supernatants were subjected to anionexchange and sizing chromatographies on Mono-Q and Superose 6-12 FPLC columns (Pharmacia-LKB Biotechnology Inc.), as described $[3,6]$. Some samples were also subjected to affinity chromatography on Con A-Sepharose [6].

Immunoabsorption. T. rangeli lysates were incubated with increasing volumes of protein A-agarose (Sigma) bearing antibodies against $T$. cruzi trypomastigote TS. The following antibodies were bound to different batches of protein A-agarose: monoclonal antibody 39 [27], rabbit IgG against purified TS, rabbit IgG against a synthetic peptide corresponding to the first 19 amino-terminal amino acid residues of TS [11], and rabbit IgG against the repeat

\section{TABLE I}

Comparison of trans-sialidase and sialidase activities of $T$. rangeli, T. cruzi and $T$. bruce $i$

\begin{tabular}{|c|c|c|c|c|}
\hline \multirow[t]{2}{*}{ Parasite species (strain /stage) } & \multirow[t]{2}{*}{ Enzyme source } & \multirow[t]{2}{*}{ Amounts $^{\mathrm{a}}$} & \multicolumn{2}{|c|}{ Enzymatic activity } \\
\hline & & & Trans-sialidase $^{\mathrm{b}}$ & Sialidase $^{c}$ \\
\hline T. rangeli $(\mathrm{SC}-58)$ & $\begin{array}{l}\text { Lysate } \\
\text { Supernatant }\end{array}$ & $\begin{array}{c}5 \times 10^{5} \\
10^{7} \\
1 \\
30\end{array}$ & $\begin{array}{c}9^{d} \\
43 \\
-4 \\
3\end{array}$ & $\begin{array}{c}718^{\mathrm{e}} \\
\mathrm{ND}^{\mathrm{f}} \\
3,503 \\
\mathrm{ND}\end{array}$ \\
\hline T. rangeli (ATCC 30032) & $\begin{array}{l}\text { Lysate } \\
\text { Supernatant }\end{array}$ & $\begin{array}{c}5 \times 10^{5} \\
10^{7} \\
1 \\
30\end{array}$ & $\begin{array}{r}0 \\
-23 \\
-14 \\
-1\end{array}$ & $\begin{array}{c}1,788 \\
\text { ND } \\
7,204 \\
\text { ND }\end{array}$ \\
\hline$T$. cruzi (trypomastigotes) & Lysate & $\begin{array}{l}10^{4} \\
10^{5} \\
5 \times 10^{5}\end{array}$ & $\begin{array}{r}215 \\
986 \\
6,550\end{array}$ & $\begin{array}{c}2 \\
418\end{array}$ \\
\hline T. cruzi (epimastigotes) & Lysate & $5 \underset{10^{5}}{\times 10^{5}}$ & $\begin{array}{r}148 \\
1,036 \\
10,731\end{array}$ & $\begin{array}{r}0 \\
32 \\
696\end{array}$ \\
\hline T. brucei (procyclics) & Lysate & $5 \times 10^{5}$ & 3,429 & 473 \\
\hline
\end{tabular}

${ }^{\text {a }}$ Total numbers of lysed parasites in lysate samples or volumes in $\mu$ l of supernatants from liver-infusion tryptose cultures containing $2.8 \times 10^{7}-3 \times 10^{7}$ parasites $/ \mathrm{ml}$.

${ }^{b}$ Trans-sialidase activity was measured by incubating the indicated amounts of supernatant or lysates with $1 \mathrm{mM}(\alpha 2$ 3)sialyllactose and $7.2 \mu \mathrm{M}$ radiolabelled lactose, in $50 \mu \mathrm{l}$ of $50 \mathrm{mM}$ Hepes, $\mathrm{pH} 7$, for 50 min. at room temperature, as described in the text. The radiolabeled sialyllactose formed was separated by anion-exchange chromatography and quantified in a beta-counter.

${ }^{\mathrm{c}}$ Sialidase activity was assayed by incubating the lysates or supernatants with $1 \mathrm{mM}$ 4-methylumbelliferyl-n-acetylneuraminic acid in $50 \mu \mathrm{l}$ of $50 \mathrm{mM}$ HEPES buffer, $\mathrm{pH} 6.6$, for $2 \mathrm{~h}$ at room temperature. The amount of fluorescent 4-methylumbelliferone formed was measured at $420 \mathrm{~nm}$ using excitation at $365 \mathrm{~nm}$ in a Titertek Fluoroskan II (Flow Laboratories Inc.).

${ }^{\mathrm{d}}$ Mean cpm of duplicates; the background value (109-136 cpm, obtained in the absence of trans-sialisase) was subtracted. Variation between duplicate values was less than $12 \%$ of the mean.

e Mean number of fluorescence units of duplicates; the background value (653-1144 fluorescence units, obtained in the absence of sialidase) was subtracted. Variation between duplicate values was less than $17 \%$ of the mean.

fot done. 
sequences from the carboxy-terminal half of TS $[4,5]$. The antibodies against synthetic peptides were from rabbits immunized with peptides coupled to keyhole limpet hemocyanin (Sigma) [11].

\section{Results and Discussion}

Using sialyl( $(\alpha 2-3)$ lactose as a sialic acid donor and lactose as the acceptor, no TS activity was detected in extracts or culture supernatants from two different strains of $T$. rangeli. The $T$. rangeli lysates contained 100 to 1000 times more lysed parasites than the TSpositive $T$. cruzi lysates which were simultaneously tested as positive controls (Table I). It is unlikely that $T$. rangeli contains a TS differing in specificity from the $T$. cruzi and $T$. bruce $i$ enzymes $[7,8,11]$, since no TS activity was detected when we used other potential sialic acid donors, i.e., sialyl $(\alpha 2-6)$ lactose, $\operatorname{poly}(\alpha 28) \mathrm{N}$-acetylneuraminic acid, fetuin or 4-methylumbelliferyl- $N$-acetylneuraminic acid (data not shown).

Although $T$. rangeli lysates and supernatants lacked TS activity, they had more sialidase activity than samples of $T$. cruzi and

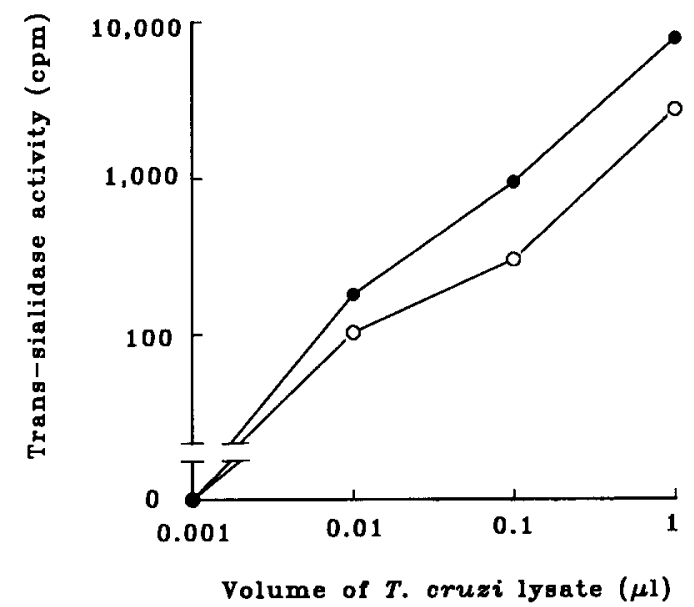

Fig. 1. T. cruzi trans-sialidase activity in the presence of $T$. rangeli sialidase. Different volumes of a lysate from $T$. cruzi trypomastigotes were tested for trans-sialidase activity in the presence (O) or absence (O) of $10 \mu \mathrm{l}$ volumes of a $T$. rangeli lysate. The number of lysed $T$. cruzi or $T$. rangeli per $\mu$ l of lysate was $10^{6}$.
T. brucei lysates derived from equivalent numbers of parasites (Table I). To exclude the possibility that the high sialidase activity in $T$. rangeli hindered the detection of a TS
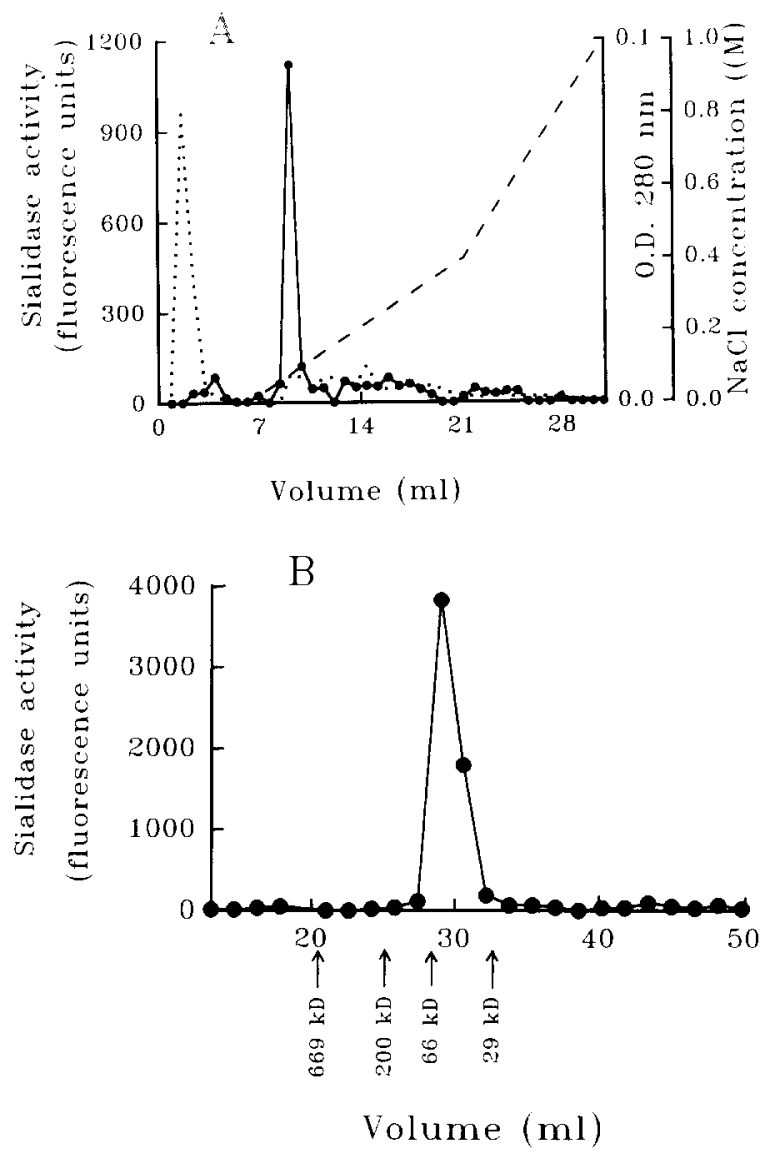

Fig. 2. Purification of sialidase activity from lysates of $T$. rangeli (ATCC 30032). Sialidase activity in the chromatographic fractions was followed by the 4-methylumbelliferyl$N$-acetylneuraminic acid assay. The results, after subtracting the background values (790-1033 fluorescence units, in the absence of sialidase), are represented by the solid lines. No trans-sialidase activity was detected in any of the fractions, using radiolabeled lactose as acceptor, and sialyl $(\alpha 2-3)$ lactose, sialyl $(\alpha 2-6)$ lactose, poly $(\alpha 2-8) N$-acetylneuraminic acid, 4-methylumbelliferyl- $N$-acetylneuraminic acid, and fetuin as donors (not shown). (A) Fractions eluted from a Mono $\mathrm{Q}$ column with an $\mathrm{NaCl}$ gradient in $20 \mathrm{mM}$ Tris $/ \mathrm{HCl}$ containing $0.1 \% \mathrm{NP}-40$. The optical densities at $280 \mathrm{~nm}$ are represented by the dotted line. The broken line represents the $\mathrm{NaCl}$ concentrations in the gradient. (B) Fractions eluted from Superose 12-Superose 6 columns run in tandem. The chromatography was performed in the presence of $0.4 \%$ bovine serum albumin and $0.1 \% \mathrm{NP}-40$ to minimize non-specific interactions with the gel matrix. The positions where protein standards are eluted, and their molecular weights are shown in the $\mathrm{x}$-axis. 
present in the same sample, a $T$. rangeli lysate was tested for its ability to inhibit $T$. cruzi TS activity. Dilutions of a $T$. cruzi lysate were mixed with a fixed volume of $T$. rangeli lysate and tested for TS activity in the presence of 4 $\mathrm{mM}$ sialyllactose in a 40 -min assay. As shown in Fig. $1,10 \mu \mathrm{l}$ of the $T$. rangeli lysate (corresponding to $10^{7}$ parasites) barely inhibited the activity of TS contained in as little as $0.01 \mu$ l of $T$. cruzi lysate (corresponding to $10^{4}$ parasites). Furthermore, when $T$. rangeli lysates were subjected to anion exchange or molecular sieving chromatographies, no TS activity was uncovered in the fractions. Instead, a single peak of sialidase activity was detected with either chromatographic procedure (Fig. 2). We conclude that culture forms of $T$. rangeli do not express a TS and that their sialidase is strictly hydrolytic, while TS activity predominates over sialidase activity in $T$. cruzi and $T$. brucei enzymes $[3,4,11]$.

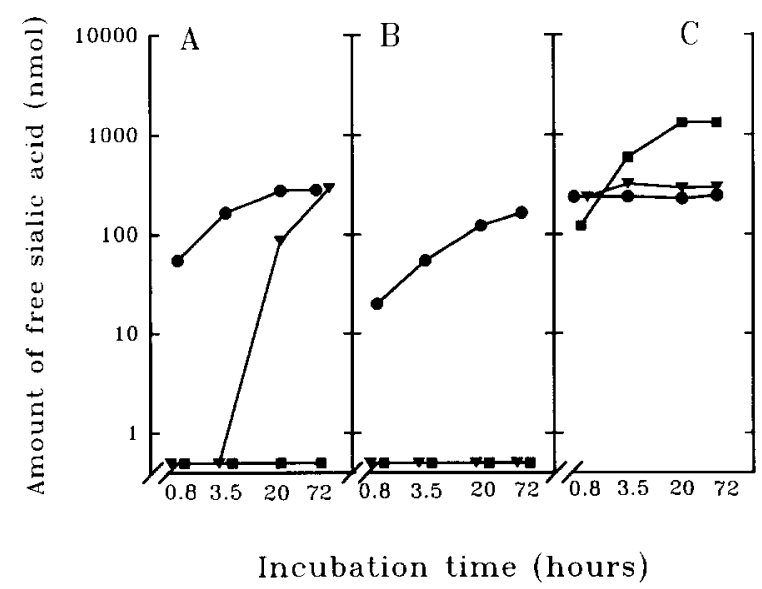

Fig. 3. Substrate specificity of the $T$. rangeli sialidase, and of the hydrolase activity of the $T$, cruzi trans-sialidase. About $300 \mathrm{nmol}$ of sialyl( $\alpha 2-3)$ lactose (circles), sialyl $(\alpha 2-$ 6)lactose (triangles), or a pentamer of $(\alpha 2-8)$-linked $N$ acetylneuraminic acid (squares) were incubated with: (A) 10 $\mu \mathrm{l}$ of a $T$. rangeli culture supernatant, pre-dialyzed against $0.15 \mathrm{M}$ phosphate-buffered saline, $\mathrm{pH} 7.2$; (B) T. cruzi TS purified by affinity chromatography on concanavalin ASepharose; or (C) $0.04 \mathrm{U}$ of $V$. cholerae sialidase. The reactions were carried out in a total volume of $50 \mu 150 \mathrm{mM}$ MES, pH 5.5, to assay for the $T$. rangeli and $V$. cholerae sialidases, or in $50 \mathrm{mM}$ HEPES, $\mathrm{pH} \mathrm{6.6,} \mathrm{to} \mathrm{assay} \mathrm{for} \mathrm{the} T$. cruzi sialidase activity). After different incubation times, the amount of free sialic acid present in $7 \mu \mathrm{l}$ of the reaction mixture was measured by the thiobarbituric method, and expressed as nmols/total reaction volume.
On the basis of its elution pattern from Superose columns, the $T$. rangeli sialidase has a molecular weight of approximately $58 \mathrm{kDa}$, a result which is in agreement with the findings of Pereira and Moss [1]. In contrast, the $T$. cruzi trypomastigote TS is a multimer of about $700 \mathrm{kDa}$, with subunits varying from $120-240$ $\mathrm{kDa}[3]$, and the T. cruzi epimastigote TS has a molecular weight of 90 [28]. The sialidase of $T$. rangeli could be further purified by affinity chromatography on Con A-Sepharose, following elution with an excess of $\alpha$-methylmannopyranoside (not shown). In this respect it resembles the TSs of T. brucei [11], T. cruzi trypomastigotes [6] and $T$. cruzi epimastigotes [28]. As might have been predicted from the high sialidase content and lack of TS activity in the supernatants of $T$. rangeli cultures, the parasites did not contain sialic acid, as measured in this paper by the HPLC-thiobarbituric acid method (not shown). This confirms previously reported data obtained with the less sensitive thiobarbituric acid method [29].

In the next series of experiments we compared the specifities of the sialidases from the different species of trypanosomes. Extracts of parasites were incubated for different periods of time with $300 \mathrm{nmol}$ of various sialylated saccharides. After $3.5 \mathrm{~h}$ of incubation with $6 \mathrm{mM}$ sialyl $(\alpha 2-3)$ lactose, the $T$. cruzi TS, $T$. rangeli and $V$. cholerae sialidases released about 55, 165 and $240 \mathrm{nmol}$ of sialic acid respectively. Neither the $T$. cruzi nor the $T$. rangeli enzymes hydrolysed $\operatorname{poly}(\alpha 2-8) N$ acetylneuraminic acid or sialyl $(\alpha 2-6)$ lactose during the first hours of incubation. Nevertheless, after $20 \mathrm{~h}$, the $T$. rangeli sialidase had hydrolysed $86 \mathrm{nM}$ of sialyl $(\alpha 2-6)$ lactose. Although we detected by thin-layer chromatography small amounts of contaminant sialyl $(\alpha 2-3)$ lactose in the sialyl $(\alpha 2-6)$ lactose preparation, this could not account for the large amounts of sialic acid released by the $T$. rangeli sialidase. These results are in agreement with Reuter and collaborators' finding that the $T$. rangeli sialidase preferentially cleaves sialyl $(\alpha 2-3)$ lactose in relation to $\operatorname{sialyl}(\alpha 2-$ 6)lactose [30]. On the other hand, the T. cruzi TS did not hydrolyse sialyl( $\alpha 2-6)$ lactose, even 


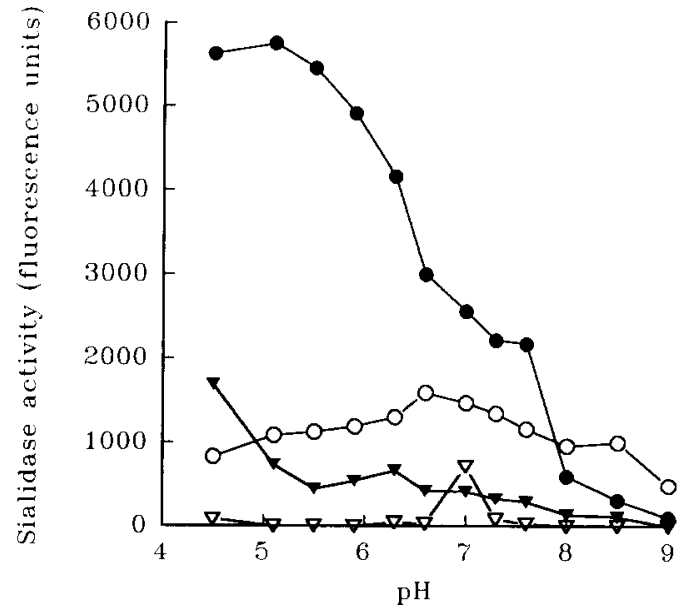

Fig. 4. Optimum pH activity of the $T$. rangeli and the $T$. cruzi sialidases. Samples of $T$. rangeli culture supernatant (closed circles), $T$. rangeli lysate (closed triangles), T. cruzi trypomastigote TS, partially purified by affinity chromatography on Con A-Sepharose (open circles), or $T$. cruzi epimastigote lysate (open triangles) were tested for sialidase activity in the 4-methylumbelliferylneuraminic acid assay. The enzymes were diluted in $50 \mathrm{mM}$ of the following buffers: acetate, $\mathrm{pH}$ 4.5; MES, $\mathrm{pH}$ 5.1-6.3; HEPES, pH 6.6-7.3; and Tris, pH 8.0-9.0.

after $72 \mathrm{~h}$ of incubation. However, it appears that at high concentrations sialyl $(\alpha 2-6)$ lactosamine can donate sialic acid to lactose in a reaction catalysed by the $T$. cruzi TS [6].

Although the substrate specificities of the $T$. cruzi and $T$. rangeli enzymes are similar, the optimum $\mathrm{pH}$ 's for their hydrolytic activities differ somewhat. Both enzymes are active over broad $\mathrm{pH}$ ranges, but the $T$. rangeli sialidase is more efficient at lower $\mathrm{pH}$ (around 5), as also reported by Pereira and Moss [1]. The TSs of $T$. cruzi trypomastigotes and epimastigotes are more active at neutral pH (Fig. 4).

The $T$. rangeli and $T$. cruzi trypomastigote enzymes are antigenically distinct. We could not deplete $T$. rangeli sialidase activity from culture supernatants by incubation with agarose-protein A bearing monoclonal and polyclonal antibodies to $T$. cruzi trypomastigote TS. The polyclonal antibodies were against the repeats and the $\mathrm{N}$-terminal region of TS $[5,11]$.

The functions of the $T$. cruzi epimastigote TS and of the $T$. rangeli sialidase are not known. One possibility is that they have specific roles in the development of the parasites in the insect vector. For example, sialic acid and/or lectins on the epithelial lining of the intestinal tract of the insect may influence the migration and/or attachment of the parasites. Whether the hematophagous vectors of these parasites synthesize sialic acid is not known, but if the appropriate saccharide acceptors are available the $T$. cruzi TS may sialylate them using sialic acid from the glycoconjugates in the ingested blood.

Although the $T$. cruzi and $T$. rangeli enzymes are antigenically distinct, they are structurally related (D. Eichinger, in preparation), and, as shown here, their substrates specificities are very similar. It is conceivable that a limited number of mutations in the $T$. rangeli sialidase will transform it into a TS. If this hypothesis is correct this could have obvious implications for the understanding of the origin and evolution of parasitism. Although clearly speculative, this idea can be tested experimentally. One prediction is that the $T$. rangeli sialidase can be converted into a TS by site-directed mutagenesis. In addition, following transfection of TS into $T$. rangeli, the parasites should adhere to mammalian cells, provided that the enzyme is expressed on the plasma membrane.

While the present studies were being prepared for publication, an abstract reporting the inability of the $T$. rangeli sialidase to act as a trans-sialidase was brought to our attention (M. Engstler, G. Reuter and R. Schauer, Biol. Chem. Hoppe-Seyler, 373, 843, 1992).

\section{Acknowledgements}

We thank Ching Huang for expert technical assistance, and Rocio Farfan for instruction and assistance in HPLC analysis. This work was supported by grants from the MacArthur Foundation, the National Institute of Health (A1 32966-01), the Rockefeller Foundation, the UNDP/World Bank/WHO Special Program for Research and Training in Tropical Diseases, and the Secretaria de Ciência e Tecnologia - Programa RHAE (Brazil). 


\section{References}

1 Pereira, M.E. (1983) A developmentally regulated neuraminidase activity in Trypanosoma cruzi. Science 219, 1444-1446.

2 Pereira, M.E. and Moss, D. (1985) Neuraminidase activity in Trypanosoma rangeli. Mol. Biochem. Parasitol. 15, 95-103.

3 Schenkman, S., Pontes de Carvalho, L. and Nussenzweig, V. (1992) Trypanosoma cruzi trans-sialidase and neuraminidase activities can be mediated by the same enzymes. J. Exp. Med. 175, 567-575.

4 Parodi, A.J., Pollevick, G.D., Mautner, M., Buschiazzo, A., Sanchez, D.O. and Frasch, A.C. (1992) Identification of the gene(s) coding for the transsialidase of Trypanosoma cruzi. EMBO J. 11, 17051710.

5 Uemura, H., Schenkman, S., Nussenzweig, V. and Eichinger, D. (1992) Only some members of a gene family in Trypanosoma cruzi encode proteins which express both trans-sialidase and neuraminidase activities. EMBO J. 11, 3837-3844.

6 Schenkman, S., Jiang, M.S., Hart, G.W. and Nussenzweig, V. (1991) A novel cell surface trans-sialidase of Trypanosoma cruzi generates a stage-specific epitope required for invasion of mammalian cells. Cell 65 , $1117-1125$.

7 Vandekerckhove, F., Schenkman, S., Pontes de Carvalho, L.C., Tomlinson, S., Kiso, M., Yoshida, M., Hasegawa, A. and Nussenzweig, V. (1992) Substratespecificity of the Trypanosoma cruzi trans-sialidase. Glycobiology 2, 541-548.

8 Ferrero-Garcia, M.A., Trombetta, S.E., Sánchez, D.O., Reglero, A., Frasch, A.C.C. and Parodi, A.J. (1993) The action of Trypanosoma cruzi trans-sialidase on glycolipids and glycoproteins. Eur. J. Biochem., in press.

9 Cazzulo, J.J. and Frasch, A.C.C. (1992) SAPA-transsialidase and cruzipain: two antigens from Trypanosoma cruzi contain immunodominant but enzymatically inactive domains. FASEB J. 6, 3258-3264.

10 Schenkman, S., Eichinger, D. (1993) Trypanosoma cruzi and cell invasion. Parasitol. Today, in press.

11 Pontes de Carvalho, L.C., Tomlinson, S., Vandekerckhove, F., Bienen, E.J., Clarkson, A.B., Jiang, M.-S., Hart, G.W. and Nussenzweig, V. (1993) Characterization of a novel trans-sialidase of Trypanosoma brucei procyclic trypomastigotes and identification of procyclin as a sialic acid acceptor. J. Exp. Med. 177, 465-474.

12 Schenkman, R.P.F., Vandekerckhove, F. and Schenkman, S. (1993) Mammalian cell sialic acid enhances invasion by Trypanosoma cruzi. Infect. Immun. 61, 898902.

13 Ming, M., Chuenkova, M., Ortega-Barria, E. and Pereira, M.E.A. (1993) Mediation of Trypanosoma cruzi invasion by sialic acid on the host cell and transsialidase on the trypanosome. Mol. Biochem. Parasitol. $59,243-252$

14 Hall, B.F., Webster, P., Ma, A.K., Joiner, K.A. and Andrews, N.W. (1992) Desialylation of lysosomal membrane glycoproteins by Trypanosoma cruzi: a role for the surface neuraminidase in facilitating parasite entry into the host cell cytoplasm. J. Exp. Med. 176, 313-325.
15 Afchain, D., Le Ray, D., Fruit, J. and Capron, A. (1979) Antigenic make-up of Trypanosoma cruzi culture forms: identification of a specific component. $J$. Parasitol. 65, 507-514.

16 Grogl, M. and Kuhn, R.E. (1984) Identification of antigens of culture forms of Trypanosoma cruzi and Trypanosoma rangeli recognized by sera from patients with chronic Chagas' disease. J. Parasitol. 70, 822-824.

17 Brener, Z. (1973) Biology of Trypanosoma cruzi. Annu.Rev.Microbiol. 27, 347-382.

18 Vickerman, K. (1985) Developmental cycles and biology of pathogenic trypanosomes. Br. Med. Bull. $41,105-114$.

19 D'Alessandro, A. (1976) Biology of Trypanosoma rangeli. In: The Biology of the Kinetoplastida (Lumsden, W.H.R. and Evans, D.A., eds.), pp. 327-403. Academic Press, New York.

20 Steindel, M., Pinto, J.C., Toma, H.K., Mangia, R.H., Ribeiro-Rodrigues, R. and Romanha, A.J. (1991) Trypanosoma rangeli (Tejera, 1920) isolated from a sylvatic rodent (Echimys dasythrix) in Santa Catarina Island, Santa Catarina State: first report of this trypanosome in southern Brazil. Mem. Inst. Oswaldo Cruz 86, 73-79.

21 Camargo, E.P. (1964) Growth and differentiation in Trypanosoma cruzi: Origin of metacyclic trypomastigotes in liquid media. Rev. Inst. Med. Trop. Sao Paulo. $6,93-100$.

22 Silva, L.H.P. and Nussenzweig, V. (1953) Sobre uma cepa de Trypanosoma cruzi altamente virulenta para o camundongo branco. Folia Clin. Biol. 20, 191-203.

23 Jennings, F.W., Urquhart, G.M., Murray, P.K. and Miller, B.M. (1983) Treatment with suramin and 2substituted 5-nitroimidazoles of chronic murine Trypanosoma brucei infections with central nervous system involvement. Trans. R. Soc. Trop. Med. Hyg. 77, 693698.

24 Bienen, E.J., Hill, G.C. and Shin, K.-O. (1983) Elaboration of mitochondrial function during Trypanosoma brucei differentiation. Mol. Biochem. Parasitol. 7, 75-86.

25 Warren, L. (1959) The thiobarbituric acid assay of sialic acids. J. Biol. Chem. 234, 1971-1975.

26 Powell, L.D. and Hart, G.W. (1986) Quantitation of picomole levels of $\mathrm{N}$-acetyl and $\mathrm{N}$-glycolylneuraminic acids by a HPLC-adaptation of the thiobarbituric acid assay. Anal. Biochem. 157, 179-185.

27 Schenkman, S., Diaz, C. and Nussenzweig, V. (1991) Attachment of Trypanosoma cruzi trypomastigotes to receptors at restricted cell surface domains. Exp. Parasitol. 72, 76-86.

28 Chaves, L.B., Briones, M.R.S. and Schenkman, S. (1993) Trans-sialidase from Trypanosoma cruzi epimastigote is expressed at the stationary phase and is different from the enzyme expressed in trypomastigotes. Mol. Biochem. Parasitol. 61, 97-106.

29 Schottelius, J. (1984) Differentiation between Trypanosoma cruzi and Trypanosoma rangeli on the basis of their sialic acid content. Tropenmed. Parasitol. 35, 160 162

30 Reuter, G., Shauer, R., Prioli, R. and Pereira, M.A. (1987) Isolation and properties of a sialidase from Trypanosoma rangeli. Glycoconjugate 4, 339-348. 